\title{
A Class of Optimal Portfolio Liquidation Problems with a Linear Decreasing Impact
}

\author{
Jiangming Ma, Zheng Yin, and Hongjing Chen \\ School of Economic Mathematics, Southwestern University of Finance and Economics, Chengdu, Sichuan 611130, China \\ Correspondence should be addressed to Jiangming Ma; mjm121121@163.com
}

Received 21 February 2017; Accepted 22 March 2017; Published 26 April 2017

Academic Editor: Honglei Xu

Copyright (C) 2017 Jiangming Ma et al. This is an open access article distributed under the Creative Commons Attribution License, which permits unrestricted use, distribution, and reproduction in any medium, provided the original work is properly cited.

\begin{abstract}
A problem of an optimal liquidation is investigated by using the Almgren-Chriss market impact model on the background that the $n$ agents liquidate assets completely. The impact of market is divided into three components: unaffected price process, permanent impact, and temporary impact. The key element is that the variable temporary market impact is analyzed. When the temporary market impact is decreasing linearly, the optimal problem is described by a Nash equilibrium in finite time horizon. The stochastic component of the price process is eliminated from the mean-variance. Mathematically, the Nash equilibrium is considered as the second-order linear differential equation with variable coefficients. We prove the existence and uniqueness of solutions for the differential equation with two boundaries and find the closed-form solutions in special situations. The numerical examples and properties of the solution are given. The corresponding finance phenomenon is interpreted.
\end{abstract}

\section{Introduction}

The investor often considers optimal execution in the market of transactions within a fixed time period, optimizing some trade-off between risk and reward. Slow trading reduces transaction costs and fast liquidation decreases the volatility risk. The investors must choose an optimal strategy which could bring them the maximization of expected revenues and minimization of the variance of revenues. The meanvariance model is given by Markowitz $[1,2]$ and is a pretty important position in modern portfolio theory. The model gives an optimal investment portfolio method when investors want to get the highest return specifying their acceptable risk level. Samuelson [3], Hakansson [4], and Pliska [5] extend the model from the single-period case to the multiperiod discrete-time case. Many scholars continue to make further researches in the recent decades, and the continuous-time case is studied by Cox and Huang [6], Duffie and Richardson [7], Karatzas et al. [8], Schweizer [9], and so forth. The meanvariance optimal problem becomes how to maximize the expected return of time $t$ and minimize the variance of the return of time $t$ at the same time. In the model of Almgren and Chriss [10], the market impact is divided into a linear permanent impact and temporary impact. The temporary impact only affects the trade when the trade happens but the permanent impact affects all trading process. Almgren [11] uses the mean-variance and studies the optimal execution with a nonlinear temporary market impact.

Since the model in Almgren and Chriss [10] is proposed, some scholars have investigated the optimal investment problem based on the model. Huberman and Stanzl [12] show that permanent market impact must be linear in the trade quantity and symmetry between buyers and sellers in order to rule out any price manipulation strategies. Bouchaud et al. [13] considered that market impact must be temporary and decay as a power law. However, Attari et al. [14] obtain a conclusion that the market price impact model only has temporary impact and show that the elastic market has the profitable liquidity provision. Schoeneborn and Schied [15] acquire that the illiquid market has multiplayers. They consider a case where the market has predatory trading. Carlin et al. [16] present an episodic illiquidity model when the cooperation breaks up in financial markets. Schied and Schoeneborn [17] find the dynamic optimal liquidation strategies in the illiquid market by the stochastic control approach. Carmona and Joseph Yang [18] change the permanent component 
of market price impact to solve the equilibrium of the predatory trading game and comparative statics from the HJB equation. Besides the continuous time, many scholars discuss the discrete time and the optimal strategies from the utility function maximization. Schied et al. [19] show that the utility function maximization is equal to the meanvariance maximization and the utility function maximization is simplified to maximize mean-variance. They prove that the results not only meet the continuous-time model but also satisfy the discrete-time model. Lorenz and Almgren [20] find the mean-variance optimization strategies by the means of a dynamic programming principle. At the same time, several scholars investigate the situation in which the market impact function has the decay factor. Gatheral [21] studies the properties of the market impact function with the exponential decay, power-law decay, and other situations. Except the above two decay functions, Schied [22] further discusses the capped linear decay and Gaussian decay and gives some useful properties.

Schied and Zhang [23] study $n$ risk-averse agents who trade a finance product and get the equilibrium strategies in both the finite and infinite time horizons. They give the market price process in which the coefficients of permanent and temporary price impact components are constant and prove the existence and uniqueness of Nash equilibria.

Based on the models of Almgren and Chriss [10] and Schied and Zhang [23], we discuss a case where the coefficient of temporary market impact decreases linearly in a short time. This case is different from those presented in [10, 23]. There exists a unique Nash equilibrium in the market. The existence and uniqueness of the Nash equilibria are equivalent to the existence and uniqueness of solutions for the second differential equation with variable coefficients. The concrete form of optimal strategy in special cases is given. We discuss the various strategies in which the volatility and temporary impact of market change and get some interesting phenomena.

The paper is organized as follows. In Section 2, we state the optimal investment in the Almgren-Chriss framework and drive the equation of market impact price, the return function, and the optimal objective function. In Section 3, we give the system of the Nash equilibrium and prove the existence and the uniqueness. When the market has two agents, the optimal solution is given. In Section 4, the numerical examples and the corresponding financial interpretations are illustrated.

\section{Statement of Background}

2.1. Introduction of Models. In this paper, we use the model which is a continuous-time framework of Almgren and Chriss [10]. In this framework, every investor is active in a fixed time period $[0, T]$. An investor in this framework is required to hold $x$ shares at the initial position and completely trade at the terminal time $T$. Investors know all market information. The information flow is considered as a filtration $\left(\mathscr{F}_{t}\right)_{t>0}$ on a given probability space $(\Omega, \mathscr{F}, \mathscr{P})$. The investor's trading strategy is defined by $X=(X(t))_{t \in[0, T]}$. The trading strategy satisfies the following properties: (i) $X$ is absolutely continuous and bounded with derivative $\dot{X}(t)$ and satisfies the liquidation constraint $X(T)=0$.

(ii) $X$ is adapted to the filtration $\left(\mathscr{F}_{t}\right)_{t>0}$. There is a progressively measurable process $\dot{X}(t)$ which satisfies $\int_{0}^{T}(\dot{X}(t, \omega))^{2} d t<\infty . X$ satisfies

$$
X(t, \omega)=X(0, \omega)+\int_{0}^{t} \dot{X}(s, \omega) d s, \quad t \in[0, T] .
$$

The class of all strategies is denoted by $\mathscr{X}(x, T) . \mathscr{X}(x, T)$ satisfies $X(0)=x$ for given $x \in \mathbb{R}$. The subclass of all strategies is denoted by $\mathscr{X}_{\mathrm{det}}(x, T)$. It does not depend on $\omega$ and is in $\mathscr{X}(x, T) . S^{0}$ is defined by the unaffected price process. It describes the fluctuations of asset prices which are perceived by an investor in the symmetric information market during the time interval $[0, T]$. In the Almgren-Chriss model, it is considered as the price of the risky asset which follows the Bachelier model [24]. Further, an extra drift in addition to the current model is based on the Bachelier model [24]. Therefore, we have

$$
S^{0}(t)=S_{0}+\sigma W(t)+\int_{0}^{t} b(s) d s,
$$

where $S_{0}$ is the initial price, $W(t)$ is the standard Brownian motion, the positive constant $\sigma$ is the volatility of unaffected price process, and $b(t)$ is an extra drift which is deterministic and continuous.

The investor chooses a trading strategy that is described by $X_{t}$ which is the number of shares at time $t$. The market impact is the adverse feedback effect about the quoted price of a stock caused by the investor's own trading and it is divided into three components: unaffected price process and permanent and temporary impact components. Firstly, the temporary impact shows the temporary imbalances between supply and demand caused by the trading of investors. Secondly, the permanent impact shows the change in the equilibrium due to trading which will last for the life of liquidation. Finally, in the linear Almgren-Chriss framework, the price process is assumed to be

$$
S^{X}:=S^{0}(t)+\gamma(X(t)-X(0))+k \dot{X}(t), \quad t \in[0, T],
$$

where the parameters $\gamma>0$ and $k>0$ are constants and represent permanent and temporary price impact components, respectively. The permanent impact component is a linear function of the variable share number. The temporary impact component is the linear function of trading speed. Huberman and Stanzl [12] consider that the permanent market impact must be linear in the price process in order to rule out any price manipulation strategies.

When the coefficient of temporary impact is a linearly decreasing function, the function satisfies $\rho-\lambda_{0} t$, where $\lambda_{0}>0$. We denote it by $\lambda(t)=\rho-\lambda_{0} t$, where $\lambda_{0}>0$. The price process becomes

$$
S^{X}:=S^{0}(t)+\gamma(X(t)-X(0))+\lambda(t) \dot{X}(t),
$$

$t \in[0, T]$. 
At each time $t \in[0, T]$, the infinitesimal amounts of $-\dot{X}(t) d t$ shares are sold at price $S^{X}(t)$. So the revenue of each time is the product of the amount of shares and the price. Therefore, the total revenues are the sum of revenues at each time generated by the strategy $X \in \mathscr{X}(x, T)$. It is represented by

$$
\mathfrak{R}(X):=-\int_{0}^{T} \dot{X}(t) S^{X}(t) d t .
$$

So the optimal trade execution problem becomes the maximization of expected revenues. We only need to solve the maximization of the function of expected revenues:

$$
\operatorname{maximize} \mathbb{E}[\Re(X)] \text {. }
$$

Bertsimas and Lo [25] proposed problem (6). Carmona and Joseph Yang [18] use (6) to deal with the problem of the maximization. But the volatility risk is not considered in the maximization of expected revenues. Therefore, the maximization of the mean-variance criterion is used in practice:

$$
\operatorname{maximize} \mathbb{E}[\Re(X)]-\frac{\alpha}{2} \operatorname{var}(\Re(X)),
$$

where $\alpha$ is a nonnegative risk aversion parameter. In the frame of Almgren and Chriss, problem (7) is usually discussed on the subclass $\mathscr{X}_{\text {det }}(x, T)$ of deterministic strategies (see Almgren and Chriss [10] and Almgren [11]). At the same time, some authors study the maximization of the optimal execution which is based on the utility function. The result that the maximization mean-variance of expected revenues over deterministic strategies is equivalent to the maximization of the expected utility of revenues is investigated by Schied et al. [19]:

$$
\text { maximize } \mathbb{E}\left[u_{\alpha}(\Re(X))\right]
$$

over all strategies in $\mathscr{X}(x, T)$, where

$$
u_{\alpha}:= \begin{cases}\frac{1}{\alpha}\left(1-e^{-\alpha x}\right), & \text { if } \alpha>0 \\ x, & \text { if } \alpha=0\end{cases}
$$

is a utility function with constant absolute risk aversions.

2.2. Nash Equilibrium. We suppose that all participators are active in the market. The $i_{\text {th }}$ investor uses the strategies $X_{i}$. The price process $S^{0}$ will be impacted when the investor has the strategy $X_{i}$. Following variable time $t$, the $i_{\text {th }}$ investor will be influenced by others' strategies. From (3), the market price impact model is made by the aggregated price impact; namely,

$$
\begin{array}{r}
S^{X_{1}, \ldots, X_{n}}:=S^{0}(t)+\gamma \sum_{j=1}^{n}\left(X_{j}(t)-X_{j}(0)\right)+k \sum_{j=1}^{n} \dot{X}(t), \\
t \in[0, T] .
\end{array}
$$

When the coefficient of temporary impact is $\lambda(t)=\rho-\lambda_{0} t$, the above equation becomes

$$
\begin{aligned}
S^{X_{1}, \ldots, X_{n}:=} & S^{0}(t)+\gamma \sum_{j=1}^{n}\left(X_{j}(t)-X_{j}(0)\right) \\
& +\lambda(t) \sum_{j=1}^{n} \dot{X}(t), \quad t \in[0, T] .
\end{aligned}
$$

The $i_{\text {th }}$ agent's strategy is denoted by $X_{-i}:=\left\{X_{1}, X_{i-1}, \ldots\right.$, $\left.X_{i+1}, X_{n}\right\}$. So, the revenue of $i_{\text {th }}$ investor is defined by

$$
\mathfrak{R}\left(X_{i} \mid X_{-i}\right)=-\int_{0}^{T} \dot{X}_{i}(t) S^{X_{1}, \ldots, X_{n}}(t) d t .
$$

Therefore, when every investor gets the optimal strategy, the market problem is whether there exists a Nash equilibrium.

Definition 1 (see [23]). Suppose that every agent's initial asset positions and risk aversion parameters are $x_{1}, \ldots, x_{n} \in \mathbb{R}$ and $\alpha_{1}, \ldots, \alpha_{n}$, respectively, where risk aversion parameter is nonnegative:

(a) If there exists a collection $X_{1}^{*}, \ldots, X_{n}^{*}$ of deterministic strategies such that, for each $i$ and $X_{-i}^{*}=$ $\left\{X_{1}^{*}, X_{i-1}^{*}, \ldots, X_{i+1}^{*}, X_{n}^{*}\right\}$, the strategy $X_{i}^{*} \in \mathscr{X}_{\operatorname{det}}\left(x_{i}\right.$, $T$ ) maximizes the mean-variance functional

$$
\mathbb{E}\left[\Re\left(X \mid X_{-i}^{*}\right)\right]-\frac{\alpha_{i}}{2} \operatorname{var}\left(\mathfrak{R}\left(X \mid X_{-i}^{*}\right)\right)
$$

over all $X \in \mathscr{X}_{\mathrm{det}}\left(x_{i}, T\right)$, the collection $X_{1}^{*}, \ldots, X_{n}^{*}$ is defined by a Nash equilibrium for mean-variance optimization.

(b) If there exists a collection $X_{1}^{*}, \ldots, X_{n}^{*}$ of admissible strategies such that, for each $i$, the strategy $X_{i}^{*} \in$ $\mathscr{X}\left(x_{i}, T\right)$ maximizes the expected utility

$$
\mathbb{E}\left[u_{\alpha_{i}}\left(\Re\left(X \mid X_{-i}^{*}\right)\right)\right]
$$

over all $X \in \mathscr{X}\left(x_{i}, T\right)$, the collection $X_{1}^{*}, \ldots, X_{n}^{*}$ is defined by a Nash equilibrium for CARA utility maximization.

The equilibrium strategies of utility optimization are considered as adapted when the optimal strategies satisfy the mean-variance optimization. Next, we will prove the existence and uniqueness of Nash equilibrium which are based on the mean-variance maximization. In this paper, we discuss the situation where the coefficient of temporary impact decreases linearly in a fixed time.

Definition 2 (see [26]). Let $D$ be the class of functions which is constructed by all continuously differentiable function $y(x)$ in $[a, b]$, where $y(x)$ satisfies the boundary conditions $y(a)=$ $A$ and $y(b)=B$. A function $F(x, y, \dot{y})$ which has three variables is twice continuously differentiable about every variable. In $D$, the functional $J[y(x)]$ is defined by

$$
J[y(x)]=\int_{a}^{b} F(x, y(x), \dot{y}(x)) d x .
$$


Lemma 3 (see [26]). If there is a function $y=y_{0}(x)$ in $D$, so that (15) has an extremum, the function $y=y_{0}(x)$ is the solution of the differential equation

$$
\dot{F}_{y}-\frac{d}{d x} \dot{F}_{\dot{y}}=0
$$

\section{Main Results}

The $i_{\text {th }}$ agent's admissible strategies and other strategies are denoted by $X_{i} \in \mathscr{X}\left(x_{i}, T\right)$ and $X_{-i}:=\left\{X_{1}, X_{i-1}, \ldots, X_{i+1}, X_{n}\right\}$ for $i=1, \ldots, n$, respectively. Let $Y=X_{i}(t)$; the revenue function is defined by

$$
\begin{aligned}
& \Re\left(Y \mid X_{-i}\right)=-\int_{0}^{T} \dot{Y}(t)\left[S_{0}+\sigma W(t)+\int_{0}^{t} b(s) d s\right. \\
& \left.+\gamma \sum_{j=1}^{n}\left(X_{j}(t)-X_{j}(0)\right)+\lambda(t) \sum_{j=1}^{n} \dot{X}_{j}(t)\right] d t \\
& =-\int_{0}^{T} \dot{Y}(t) S_{0} d t-\int_{0}^{T} \dot{Y}(t) \sigma W(t) d t-\int_{0}^{T} \dot{Y}(t) \\
& \cdot \int_{0}^{t} b(s) d s d t-\gamma \int_{0}^{T} \dot{Y}(t) \\
& \cdot \sum_{j=1}^{n}\left(X_{j}(t)-X_{j}(0)\right) d t-\int_{0}^{T} \dot{Y}(t) \lambda(t) \\
& \cdot \sum_{j=1}^{n} \dot{X}_{j}(t) d t=S_{0} y+\sigma \int_{0}^{T} Y(t) d W(t) \\
& +\int_{0}^{T} Y(t) b(t) d t+\gamma \sum_{j \neq i}^{n} \int_{0}^{T} Y(t) \dot{X}_{j}(t) d t+\frac{1}{2} \\
& \cdot \gamma y^{2}-\gamma y^{2}-\sum_{j \neq i}^{n} \int_{0}^{T} \lambda(t) \dot{Y}(t) \dot{X}_{j}(t) d t-\int_{0}^{T} \lambda(t) \\
& \cdot \dot{Y}^{2}(t) d t .
\end{aligned}
$$

The mean and variance of revenue function are

$$
\begin{aligned}
\mathbb{E}\left(\Re\left(Y \mid X_{-i}\right)\right)= & S_{0} y-\frac{1}{2} \gamma y^{2}+\int_{0}^{T} Y(t) b(t) d t \\
& +\gamma \sum_{j \neq i}^{n} \int_{0}^{T} Y(t) \dot{X}_{j}(t) d t \\
& -\sum_{j \neq i}^{n} \int_{0}^{T} \lambda(t) \dot{Y}(t) \dot{X}_{j}(t) d t \\
& -\int_{0}^{T} \lambda(t) \dot{Y}^{2}(t) d t, \\
\operatorname{var}\left(\Re\left(Y \mid X_{-i}\right)\right)= & \sigma^{2} \int_{0}^{T} Y^{2} d t,
\end{aligned}
$$

respectively. From Definition 1, the mean-variance function is

$$
\begin{aligned}
& \Re\left(Y \mid X_{-i}\right)-\frac{\alpha_{i}}{2} \operatorname{var}\left(\Re\left(Y \mid X_{-i}\right)\right)=S_{0} y-\frac{1}{2} \gamma y^{2} \\
& +\int_{0}^{T} Y(t) b(t) d t+\gamma \sum_{j \neq i}^{n} \int_{0}^{T} Y(t) \dot{X}_{j}(t) d t \\
& \quad-\sum_{j \neq i}^{n} \int_{0}^{T} \lambda(t) \dot{Y}(t) \dot{X}_{j}(t) d t-\int_{0}^{T} \lambda(t) \dot{Y}^{2}(t) d t \\
& \quad-\frac{\alpha_{i}}{2} \sigma^{2} \int_{0}^{T} Y^{2}(t) d t=S_{0} y-\frac{1}{2} \gamma y^{2} \\
& +\int_{0}^{T}\left[Y(t) b(t)+\gamma \sum_{j \neq i}^{n} Y(t) \dot{X}_{j}(t)\right. \\
& \left.\quad-\sum_{j \neq i}^{n} \lambda(t) \dot{Y}(t) \dot{X}_{j}(t)-\lambda(t) \dot{Y}^{2}(t)-\frac{\alpha_{i} \sigma^{2}}{2} Y^{2}(t)\right] .
\end{aligned}
$$

When all agents' strategies are deterministic, one has

$$
\begin{aligned}
& \Re\left(Y \mid X_{-i}\right)-\frac{\alpha_{i}}{2} \operatorname{var}\left(\Re\left(Y \mid X_{-i}\right)\right) \\
& \quad=c+\int_{0}^{T} L^{i}\left(t, Y(t), \dot{Y}(t) \mid X_{-i}\right) d t,
\end{aligned}
$$

where $c=S_{0} y-(1 / 2) \gamma y^{2}$ and the Lagrangian $L^{i}$ is given by

$$
\begin{aligned}
L^{i}(t, & \left.Y(t), \dot{Y}(t) \mid X_{-i}\right) \\
= & Y(t)\left(b(t)+\gamma \sum_{j \neq i}^{n} \dot{X}_{j}(t)\right)-\frac{\alpha_{i} \sigma^{2}}{2} Y^{2}(t) \\
& -\lambda(t)\left(\sum_{j \neq i}^{n} \dot{Y}(t) \dot{X}_{j}(t)+\dot{Y}^{2}(t)\right) .
\end{aligned}
$$

Theorem 4. For $n \in \mathbb{N}, \alpha_{1}, \ldots, \alpha_{n} \geqslant 0$, and $x_{n}, \ldots, x_{n}$, there exists a unique Nash equilibrium $X_{1}^{*}, \ldots, X_{n}^{*}$ for meanvariance optimization. The equilibrium strategy is the unique solution of the following second-order system of differential equation:

$$
\begin{gathered}
\alpha_{i} \sigma^{2} Y(t)-2 \dot{\lambda}(t) \dot{Y}(t)-2 \lambda(t) \ddot{Y}(t) \\
=b(t)+\gamma \sum_{j \neq i}^{n} \dot{X}_{j}(t)+\dot{\lambda}(t) \sum_{j \neq i}^{n} \dot{X}_{j}(t) \\
+\lambda(t) \sum_{j \neq i}^{n} \ddot{X}_{j}(t)
\end{gathered}
$$

with two-point boundary conditions

$$
\begin{aligned}
& X_{i}(0)=x_{i}, \\
& X_{i}(T)=0
\end{aligned}
$$


Before proving Theorem 4, we give two lemmas.

Lemma 5. In the context of Theorem 4, there exists at most one Nash equilibrium for mean-variance optimization.

Proof. We prove this lemma by contradiction and assume that there exist two different Nash equilibria strategies $X_{1}^{0}, \ldots, X_{n}^{0}$ and $X_{1}^{1}, \ldots, X_{n}^{1}$. For $\beta \in[0,1]$, let $X_{i}^{\beta}:=\beta X_{i}^{1}+(1-\beta) X_{i}^{0}$ and define

$$
\begin{aligned}
& f(\beta):=\sum_{i=1}^{n} \int_{0}^{T}\left(L^{i}\left(t, X_{i}^{\beta}(t), \dot{X}_{i}^{\beta}(t) \mid X_{-i}^{0}\right)\right. \\
& \left.+L^{i}\left(t, X_{i}^{1-\beta}(t), \dot{X}_{i}^{1-\beta}(t) \mid X_{-i}\right)\right) .
\end{aligned}
$$

Assume that maximization of the functional $Y \mapsto \int_{0}^{T} L^{i}(t$, $\left.Y(t), \dot{Y}(t) \mid X_{-i}\right) d t$ is given by $X_{i}^{k}$ for $k=0,1$. Therefore, we get $f(\beta) \leqslant f(0)$ for $\beta>0$, which implies

$$
\left.\frac{d}{d \beta}\right|_{\beta=0+} f(\beta) \leqslant 0
$$

Then, it becomes

$$
\begin{aligned}
& \left.\frac{d}{d \beta}\right|_{\beta=0+} f(\beta)=\sum_{i=1}^{n} \int_{0}^{T}\left[\gamma\left(X_{i}^{1}(t)-X_{i}^{0}(t)\right)\right. \\
& \cdot \sum_{j=1}^{n}\left(\dot{X}_{j}^{0}(t)-\dot{X}_{j}^{1}(t)\right)-\gamma\left(X_{i}^{1}(t)-X_{i}^{0}(t)\right) \\
& \cdot\left(\dot{X}_{i}^{0}(t)-\dot{X}_{i}^{1}(t)\right)+\alpha_{i} \sigma^{2}\left(X_{i}^{1}(t)-X_{i}^{0}(t)\right)^{2} \\
& +\lambda(t)\left(\dot{X}_{i}^{1}(t)-\dot{X}_{i}^{0}(t)\right) \sum_{j=1}^{n}\left(\dot{X}_{j}^{1}(t)-\dot{X}_{j}^{0}(t)\right) \\
& \left.+\lambda(t)\left(\dot{X}_{i}^{0}(t)-\dot{X}_{i}^{1}(t)\right)\right] d t .
\end{aligned}
$$

Since

$$
\begin{aligned}
& \int_{0}^{T}\left(X_{i}^{1}(t)-X_{i}^{0}(t)\right)\left(\dot{X}_{i}^{0}(t)-\dot{X}_{i}^{1}(t)\right) d t \\
& \quad=\frac{1}{2}\left(X_{i}^{1}(T)-X_{i}^{0}(T)\right)^{2}-\frac{1}{2}\left(X_{i}^{1}(0)-X_{i}^{0}(0)\right)^{2} \\
& \quad=0 \\
& \int_{0}^{T}\left(X_{i}^{1}(t)-X_{i}^{0}(t)\right)\left(\dot{X}_{j}^{0}(t)-\dot{X}_{j}^{1}(t)\right) d t \\
& \quad=-\int_{0}^{T}\left(X_{j}^{1}(t)-X_{j}^{0}(t)\right)\left(\dot{X}_{i}^{0}(t)-\dot{X}_{i}^{1}(t)\right), \\
& \sum_{i=1}^{n} \sum_{j=1}^{n} \int_{0}^{T}\left(X_{i}^{1}(t)-X_{i}^{0}(t)\right)\left(\dot{X}_{j}^{0}(t)-\dot{X}_{j}^{1}(t)\right) d t=0,
\end{aligned}
$$

we get

$$
\begin{aligned}
& \left.\frac{d}{d \beta}\right|_{\beta=0+} f(\beta)=\int_{0}^{T}\left[\alpha_{i} \sigma^{2} \sum_{i=1}^{n}\left(X_{i}^{1}(t)-X_{i}^{0}(t)\right)^{2}\right. \\
& +\lambda(t) \sum_{i=1}^{n}\left(\dot{X}_{i}^{0}(t)-\dot{X}_{i}^{1}(t)\right)^{2} \\
& \left.+\lambda(t)\left(\sum_{i=1}^{n}\left(X_{i}^{1}(t)-X_{i}^{0}(t)\right)\right)^{2}\right] d t>0,
\end{aligned}
$$

which contradicts (25).

Lemma 6. There exists at most one maximizer in $\mathscr{X}_{\mathrm{det}}(y, T)$ of the function $\int_{0}^{T} L^{i}\left(t, Y(t), \dot{Y}(t) \mid X_{-i}\right) d t$ for $i=1, \ldots, n$. If $X_{1}, \ldots, X_{n} \in C^{2}[0, T]$ exist, the equilibrium strategy is the unique solution of the two-point boundary value problem

$$
\begin{aligned}
& \alpha_{i} \sigma Y(t)-2 \dot{\lambda}(t) \dot{Y}(t)-2 \lambda(t) \ddot{Y}(t) \\
& =b(t)+\gamma \sum_{j \neq i}^{n} \dot{X}_{j}(t)+\dot{\lambda}(t) \sum_{j \neq i}^{n} \dot{X}_{j}(t) \\
& \quad+\lambda(t) \sum_{j \neq i}^{n} \ddot{X}_{j}(t), \\
& Y(0)=y, \\
& Y(T)=0 .
\end{aligned}
$$

Proof. From the strict concavity of the Lagrangian $L^{i}$ and the convexity of the set $\mathscr{X}_{\text {det }}(y, T)$, there exists at most one maximizer in $\mathscr{X}_{\mathrm{det}}(y, T)$.

Next, the existence of a maximizer under the additional assumption $X_{1}, \ldots, X_{n} \in C^{2}[0, T]$ is proven. Under the assumption and from Definition 2 and Lemma 3, the Lagrangian equation becomes

$$
\begin{aligned}
& L_{q}^{i}\left(t, Y(t), \dot{Y}(t) \mid X_{-i}\right) \\
& =b(t)+\gamma \sum_{j \neq i}^{n} \dot{X}_{j}(t)-\alpha_{i} \sigma^{2} Y(t), \\
& L_{p}^{i}\left(t, Y(t), \dot{Y}(t) \mid X_{-i}\right) \\
& =-\lambda(t) \sum_{j \neq i}^{n} \dot{X}_{j}(t)-2 \lambda(t) \dot{Y}(t), \\
& \frac{d L_{p}^{i}\left(t, Y(t), \dot{Y}(t) \mid X_{-i}\right)}{d t} \\
& =-\dot{\lambda}(t) \sum_{j \neq i}^{n} \dot{X}_{j}(t)-\lambda(t) \sum_{j \neq i}^{n} \ddot{X}_{j}(t)-2 \dot{\lambda}(t) \dot{Y}(t) \\
& \quad-2 \lambda(t) \ddot{Y}(t) ;
\end{aligned}
$$


then

$$
\begin{aligned}
& \alpha_{i} \sigma^{2} Y(t)-2 \dot{\lambda}(t) \dot{Y}(t)-2 \lambda(t) \ddot{Y}(t) \\
& =b(t)+\gamma \sum_{j \neq i}^{n} \dot{X}_{j}(t)+\dot{\lambda}(t) \sum_{j \neq i}^{n} \dot{X}_{j}(t) \\
& \quad+\lambda(t) \sum_{j \neq i}^{n} \ddot{X}_{j}(t) .
\end{aligned}
$$

Let the right-hand side of (31) be represented by $f(t), \lambda(t)=$ $\rho-\lambda_{0} t$, where $\lambda(t)>0, \rho>0$, and $\lambda_{0}>0$. The homogeneous equation of (31) is

$$
\left(\rho-\lambda_{0} t\right) \ddot{Y}(t)-\lambda_{0} \dot{Y}(t)-\frac{\alpha_{i} \sigma^{2}}{2} Y(t)=0
$$

From the results of [27], the solution of the above equation is

$$
Y(t)=C_{1} Y_{1}(t)+C_{2} Y_{2}(t)
$$

where

$$
\begin{aligned}
& Y_{1}(t)=\sum_{k=0}^{\infty} \frac{\left(\alpha_{i} \sigma^{2}\left(\rho-\lambda_{0} t\right) / 2 \lambda_{0}^{2}\right)^{k}}{k ! \Gamma(k+1)}, \\
& Y_{2}(t) \\
& =-\sum_{k=0}^{\infty} \frac{\left(\alpha_{i} \sigma^{2}\left(\rho-\lambda_{0} t\right) / 2 \lambda_{0}^{2}\right)^{k}}{k ! \Gamma(k+1)} \ln \sqrt{\frac{\alpha_{i} \sigma^{2}\left(\rho-\lambda_{0} t\right)}{2 \lambda_{0}^{2}}} \\
& \quad+\frac{1}{2} \sum_{k=0}^{\infty}\left(\frac{\alpha_{i} \sigma^{2}\left(\rho-\lambda_{0} t\right)}{2 \lambda_{0}^{2}}\right)^{k} \frac{\psi(k+1)}{k !}
\end{aligned}
$$

where $\Gamma(k)$ is the gamma function. $\psi(1)=-C ; \psi(n)=$ $-C+\sum_{k=1}^{n-1} k^{-1} \cdot \psi(t)=[\ln \Gamma(t)]_{t}^{\prime}$ is the logarithmic derivative of the gamma function. $C=0.5772 \ldots$ is the Euler constant. The Wronskian determinant is $W\left(Y_{1}, Y_{2}\right)=$ $-2 / \pi \sqrt{\alpha_{i} \sigma^{2}\left(\rho-\lambda_{0} t\right) / 2 \lambda^{2}}$. So the solution of (31) can be represented by

$$
\begin{aligned}
Y(t)= & C_{1} Y_{1}+C_{2} Y_{2}+Y_{2} \int_{0}^{t} Y_{1} \frac{f(s)}{\lambda(s)} \frac{d s}{W} \\
& -Y_{1} \int_{0}^{t} Y_{2} \frac{f(s)}{\lambda(s)} \frac{d s}{W} .
\end{aligned}
$$

$C_{1}$ and $C_{2}$ in (35) are determined by the boundary conditions $Y(0)=y$ and $Y(T)=0$. Now, we prove that $Y^{*}$ is indeed the maximizer of our problem. From the concavity of (21) and the solution $Y^{*}$ of (31), we get

$$
\begin{aligned}
& L^{i}\left(t, Y^{*}(t), \dot{Y}^{*}(t) \mid X_{-i}\right)-L^{i}\left(t, Y(t), \dot{Y}(t) \mid X_{-i}\right) \\
& \quad \geqslant L_{q}^{i}\left(t, Y^{*}(t), \dot{Y}^{*}(t) \mid X_{-i}\right)\left(Y^{*}(t)-Y(t)\right) \\
& \quad+L_{p}^{i}\left(t, Y^{*}(t), \dot{Y}^{*}(t) \mid X_{-i}\right)\left(\dot{Y}^{*}(t)-\dot{Y}(t)\right) \\
& \quad=\left(\frac{d}{d t} L_{p}^{i}\left(t, Y^{*}(t), \dot{Y}^{*}(t) \mid X_{-i}\right)\right)\left(Y^{*}(t)-Y(t)\right) \\
& \cdot L_{p}^{i}\left(t, Y^{*}(t), \dot{Y}^{*}(t) \mid X_{-i}\right)\left(\dot{Y}^{*}(t)-\dot{Y}(t)\right) \\
& \quad=\frac{d}{d t}\left(L_{p}^{i}\left(t, Y^{*}(t), \dot{Y}^{*}(t) \mid X_{-i}\right)\right)\left(Y^{*}(t)-Y(t)\right) .
\end{aligned}
$$

Then, we obtain

$$
\begin{aligned}
& \int_{0}^{T} L^{i}\left(t, Y^{*}(t), \dot{Y}^{*}(t) \mid X_{-i}\right) d t \\
& \quad-\int_{0}^{T} L^{i}\left(t, Y(t), \dot{Y}(t) \mid X_{-i}\right) d t \\
& \quad \geqslant \int_{0}^{T} \frac{d}{d t}\left(L_{p}^{i}\left(t, Y^{*}(t), \dot{Y}^{*}(t) \mid X_{-i}\right)\right) \\
& \cdot\left(Y^{*}(t)-Y(t)\right) d t=0,
\end{aligned}
$$

where $Y^{*}(0)=Y(0)$ and $Y^{*}(T)=Y(T)$.

Proof of Theorem 4. From Lemma 5, it is shown that there exists at most one Nash equilibrium. Lemma 6 tells us that every Nash equilibrium strategy must be the solution of the following system:

$$
\begin{aligned}
& \alpha_{i} \sigma Y(t)-2 \dot{\lambda}(t) \dot{Y}(t)-2 \lambda(t) \ddot{Y}(t) \\
& =b(t)+\gamma \sum_{j \neq i}^{n} \dot{X}_{j}^{*}(t)+\dot{\lambda}(t) \sum_{j \neq i}^{n} \dot{X}_{j}^{*}(t) \\
& \quad+\lambda(t) \sum_{j \neq i}^{n} \ddot{X}_{j}^{*}(t) \\
& Y(0)=y \\
& Y(T)=0 .
\end{aligned}
$$

From Lemma 6, we get the solution of homogeneous equation for the above equation. Because

$$
\Delta=\left|\begin{array}{cc}
Y_{1}(0) & Y_{2}(0) \\
Y_{1}(T) & Y_{2}(T)
\end{array}\right| \neq 0
$$

the above nonhomogeneous differential equation has a unique solution. So there exists a unique Nash equilibrium $X_{1}^{*}, \ldots, X_{n}^{*}$ for mean-variance optimization.

The maximization of (20) and (21) will become the linearquadratic differential game with state constraints. We use the method of Euler-Lagrange. The problem is considered 
to prove the existence and uniqueness of solution for the second-order linear differential equation with variable coefficients and two-point boundary conditions. The existence and uniqueness of solutions are equal to the existence and uniqueness of the Nash equilibrium. We will prove that the Nash equilibrium of mean-variance optimization is the CARA utility optimization.

Corollary 7. For $n \in \mathbb{N}, \alpha_{1}, \ldots, \alpha_{n} \geqslant 0$, and $x_{1}, \ldots, x_{n}$, the Nash equilibrium of mean-variance optimization in Theorem 4 is also a Nash equilibrium for CARA utility maximization.

Proof. Suppose that the unique Nash equilibrium with meanvariance optimization in Theorem 4 is $X_{1}^{*}, \ldots, X_{n}^{*}$. When $X_{i}^{*}=\left\{X_{1}^{*}, \ldots, X_{i-1}^{*}, X_{i+1}^{*}, \ldots, X_{n}^{*}\right\}$, the agent $i$ thinks that the price process

$$
S^{X_{-i}^{*}}:=S^{0}(t)+\gamma \sum_{j=i}^{n}\left(X_{j}(t)-X_{j}(0)\right)+\lambda(t) \sum_{j=i}^{n} \dot{X}_{j}(t)
$$

is unperturbed. So, it can be simplified by

$$
S^{X_{-i}^{*}}:=S_{0}+\sigma W(t)+\int_{0}^{t} b_{i}(t) d t
$$

where $b_{i}(t)$ is a deterministic and continuous function. From the study of Schied et al. [19], the unperturbed price process $S^{X_{-i}^{*}}$ has all exponential moments; that is, $\mathbb{E}\left[e^{\beta S^{X_{-i}^{*}}}\right]<\infty$ for all $\beta \in \mathbb{R}$. Under the above assumption and the given positive constant $\alpha_{i}$, we have

$$
\begin{aligned}
& \sup _{X \in \mathscr{X}\left(x_{i}, T\right)} \mathbb{E}\left[u_{\alpha_{i}}\left(\Re\left(X_{i} \mid X_{-i}\right)\right)\right] \\
& =\sup _{X \in \mathscr{X}_{\mathrm{det}}\left(x_{i}, T\right)} \mathbb{E}\left[u_{\alpha_{i}}\left(\Re\left(X_{i} \mid X_{-i}\right)\right)\right] .
\end{aligned}
$$

From Theorem 4 , the optimal strategy $X_{-i}^{*}$ must be deterministic and unique as long as it exists. We get that the strategy $X_{i}^{*} \in \mathscr{X}_{\mathrm{det}}\left(x_{i}, T\right)$ satisfies

$$
\begin{aligned}
\mathbb{E} & {\left[u_{\alpha_{i}}\left(\Re\left(X_{i} \mid X_{-i}\right)\right)\right] } \\
& =\frac{1}{\alpha_{i}}\left(1-e^{-\alpha_{i} \mathbb{E}\left(\Re\left(X_{i} \mid X_{-i}\right)\right)+\left(\alpha_{i} / 2\right) \operatorname{var}\left(\Re\left(X_{i} \mid X_{-i}\right)\right)}\right) .
\end{aligned}
$$

It means that the maximization of CARA utility is equivalent to maximization of the mean-variance function. That is, the problem of optimal CARA utility can be simplified by the mean-variance optimization. When $\alpha_{i}=0$, the result is also right.

Let $\left(\widetilde{\mathscr{F}}_{t}\right)_{t \geqslant 0}$ be any subfiltration of $\left(\mathscr{F}_{t}\right)_{t \geqslant 0}$. From the proof of Corollary 7, we get that the Nash equilibrium for meanvariance optimization in Theorem 4 is also a Nash equilibrium for CARA utility. The mean-variance optimization is equivalent to the maximization of CARA utility. At the same time, the optimal strategies are adapted to $\left(\widetilde{\mathscr{F}}_{t}\right)_{t \geqslant 0}$.

We will simplify the model based on the fact that all agents have the same risk aversion.
Corollary 8. Under assumption of Theorem 4, suppose that $\alpha_{1}=\cdots=\alpha_{n}=\alpha \geqslant 0$. Then

$$
\sum(t):=\sum_{i=1}^{n} X_{i}^{*}(t)
$$

is the unique solution of the following differential equation with two-point boundary value problem:

$$
\begin{aligned}
& \alpha \sigma^{2} \sum(t)+(-(n+1) \dot{\lambda}(t)+(1-n) \gamma) \dot{\sum}(t) \\
& \quad-(n+1) \lambda(t) \ddot{\sum}(t)=n b(t), \\
& \sum(0)=\sum_{i}^{n}\left(x_{i}\right), \\
& \sum(T)=0 .
\end{aligned}
$$

For $\sum(t)$, each equilibrium strategy $X_{i}^{*}$ is equal to the unique solution of the following differential equation with two-point boundary value problem:

$$
\begin{aligned}
& \alpha \sigma^{2} Y(t)-\dot{\lambda}(t) \dot{Y}(t)+\gamma \dot{Y}(t)-\lambda(t) \ddot{Y}(t) \\
& \quad=b(t)+\gamma \dot{\sum}(t)+\dot{\lambda}(t) \dot{\sum}(t)+\lambda(t) \dot{\sum}(t), \\
& X_{i}(0)=x_{i}, \\
& X_{i}(T)=0 .
\end{aligned}
$$

Proof. Let $\sum(t):=\sum_{j=1}^{n} X_{j}(t) ;(31)$ can be written in the form

$$
\begin{aligned}
& \alpha \sigma^{2} Y(t)-\dot{\lambda}(t) \dot{Y}(t)+\gamma \dot{Y}(t)-\lambda(t) \ddot{Y}(t) \\
& \quad=b(t)+\gamma \dot{\sum}(t)+\dot{\lambda}(t) \dot{\sum}(t)+\lambda(t) \ddot{\sum}(t) .
\end{aligned}
$$

Next, we discuss a special situation in which $b=0$.

Corollary 9. Under assumption of Corollary 8, additionally, suppose that $b=0$ and $\alpha>0$; the equilibrium strategy $\sum(t)$ satisfies the form

$$
\begin{gathered}
\alpha \sigma^{2} \sum(t)+(-(n+1) \dot{\lambda}(t)+(1-n) \gamma) \dot{\sum}(t) \\
-(n+1) \lambda(t) \ddot{\sum}(t)=0 .
\end{gathered}
$$

When $(n-1) \gamma /(n+1) \lambda_{0}$ is not an integer, the solution of (48) is

$$
\begin{gathered}
\sum(t)=\left(2 \sqrt{\frac{\alpha \sigma^{2}\left(\rho-\lambda_{0} t\right)}{(n+1) \lambda_{0}^{2}}}\right)^{(n-1) \gamma /(n+1) \lambda_{0} / 2} \\
\cdot\left[C_{1} i^{(n-1) \gamma /(n+1) \lambda_{0}} I_{(n-1) \gamma /(n+1) \lambda_{0}}\left(2 \sqrt{\frac{\alpha \sigma^{2}\left(\rho-\lambda_{0} t\right)}{(n+1) \lambda_{0}^{2}}}\right)\right.
\end{gathered}
$$




$$
\begin{aligned}
& +C_{2}\left(\left(i^{(n-1) \gamma /(n+1) \lambda_{0}} I_{(n-1) \gamma /(n+1) \lambda_{0}}\left(2 \sqrt{\frac{\alpha \sigma^{2}\left(\rho-\lambda_{0} t\right)}{(n+1) \lambda_{0}^{2}}}\right)\right.\right. \\
& \cdot \cos \frac{(n-1) \gamma \pi}{(n+1) \lambda_{0}}-(-1)^{(n-1) \gamma /(n+1) \lambda_{0}} \\
& \left.\cdot i^{(n-1) \gamma /(n+1) \lambda_{0}} I_{(n-1) \gamma /(n+1) \lambda_{0}}\left(2 \sqrt{\frac{\alpha \sigma^{2}\left(\rho-\lambda_{0} t\right)}{(n+1) \lambda_{0}^{2}}}\right)\right) \\
& \left.\left.\cdot\left(\sin \frac{(n-1) \gamma \pi}{(n+1) \lambda_{0}}\right)^{-1}\right)\right] .
\end{aligned}
$$$$
\begin{aligned}
& +\frac{1}{2}(-1)^{(n-1) \gamma /(n+1) \lambda_{0}} \\
& \left.\cdot \sum_{k=0}^{\infty}\left(\frac{\left(2 \sqrt{\alpha \sigma^{2}\left(\rho-\lambda_{0} t\right) /(n+1) \lambda_{0}^{2}}\right)}{2}\right)\right)^{2 k+(n-1) \gamma /(n+1) \lambda} \\
& \left.\left.\cdot \frac{\Psi(k+1)+\Psi\left((n-1) \gamma /(n+1) \lambda_{0}+k+1\right)}{k !\left((n-1) \gamma /(n+1) \lambda_{0}+k\right) !}\right]\right\}
\end{aligned}
$$

When $(n-1) \gamma /(n+1) \lambda_{0}$ is an integer, the solution of $(48)$ is

$$
\begin{aligned}
& \sum(t)=\left(2 \sqrt{\frac{\alpha \sigma^{2}\left(\rho-\lambda_{0} t\right)}{(n+1) \lambda_{0}^{2}}}\right)^{(n-1) \gamma /(n+1) \lambda_{0} / 2} \\
& \left\{C_{1} i^{(n-1) \gamma /(n+1) \lambda_{0}} I_{(n-1) \gamma /(n+1) \lambda_{0}}\left(2 \sqrt{\frac{\alpha \sigma^{2}\left(\rho-\lambda_{0} t\right)}{(n+1) \lambda_{0}^{2}}}\right)\right. \\
& +C_{2} i^{(n-1) \gamma /(n+1) \lambda_{0}} \frac{2}{\pi}\left[(-1)^{(n-1) \gamma /(n+1) \lambda_{0}+1}\right. \\
& I_{(n-1) \gamma /(n+1) \lambda_{0}}\left(2 \sqrt{\frac{\alpha \sigma^{2}\left(\rho-\lambda_{0} t\right)}{(n+1) \lambda_{0}^{2}}}\right) \\
& \cdot \ln \frac{\left(2 \sqrt{\alpha \sigma^{2}\left(\rho-\lambda_{0} t\right) /(n+1) \lambda_{0}^{2}}\right)}{2}+\frac{1}{2} \\
& \sum_{k=0}^{(n-1) \gamma /(n+1) \lambda_{0}-1} \frac{\left((n-1) \gamma /(n+1) \lambda_{0}-k-1\right) !}{k !} \\
& y=\left(2 \sqrt{\frac{\alpha \sigma^{2}\left(\rho-\lambda_{0} t\right)}{(n+1) \lambda_{0}^{2}}}\right)^{(n-1) \gamma /(n+1) \lambda_{0} / 2}\left[C_{1} i^{(n-1) \gamma /(n+1) \lambda_{0}} I_{(n-1) \gamma /(n+1) \lambda_{0}}\left(2 \sqrt{\frac{\alpha \sigma^{2}\left(\rho-\lambda_{0} t\right)}{(n+1) \lambda_{0}^{2}}}\right)+C_{2}\right. \\
& \left.\cdot \frac{i^{(n-1) \gamma /(n+1) \lambda_{0}} I_{(n-1) \gamma /(n+1) \lambda_{0}}\left(2 \sqrt{\alpha \sigma^{2}\left(\rho-\lambda_{0} t\right) /(n+1) \lambda_{0}^{2}}\right) \cos \left((n-1) \gamma \pi /(n+1) \lambda_{0}\right)-(-1)^{(n-1) \gamma /(n+1) \lambda_{0}} i^{(n-1) \gamma /(n+1) \lambda_{0}} I_{(n-1) \gamma /(n+1) \lambda_{0}}\left(2 \sqrt{\alpha \sigma^{2}\left(\rho-\lambda_{0} t\right) /(n+1) \lambda_{0}^{2}}\right)}{\sin \left((n-1) \gamma \pi /(n+1) \lambda_{0}\right)}\right] . \\
& \alpha \sigma^{2} \sum(t)+(-(n+1) \dot{\lambda}(t)+(1-n) \gamma) \dot{\sum}(t) \\
& -(n+1) \lambda(t) \ddot{\sum}(t)=0 . \\
& \left(\rho-\lambda_{0} t\right) \ddot{y}+\left[-\lambda_{0}+\frac{(n-1) \gamma}{\lambda_{0}(n+1)}\right] \dot{y}-\frac{\alpha \sigma^{2}}{\lambda_{0}(n+1)} y \\
& =0 \text {. }
\end{aligned}
$$

where $I_{\nu}(x)=\sum_{k=0}^{\infty}\left((x / 2)^{2 k+v} / k ! \Gamma(\nu+k+1)\right)$ and $\psi(x)$ is the

When $(n-1) \gamma /(n+1) \lambda_{0}$ is an integer, the solution of (52) is

$$
\begin{aligned}
y= & \left(2 \sqrt{\frac{\alpha \sigma^{2}\left(\rho-\lambda_{0} t\right)}{(n+1) \lambda_{0}^{2}}}\right)^{(n-1) \gamma /(n+1) \lambda_{0} / 2} \\
& \cdot\left\{C_{1} i^{(n-1) \gamma /(n+1) \lambda_{0}} I_{(n-1) \gamma /(n+1) \lambda_{0}}\left(2 \sqrt{\frac{\alpha \sigma^{2}\left(\rho-\lambda_{0} t\right)}{(n+1) \lambda_{0}^{2}}}\right)\right.
\end{aligned}
$$

$$
\begin{aligned}
& +C_{2} i^{(n-1) \gamma /(n+1) \lambda_{0}} \frac{2}{\pi}\left[(-1)^{(n-1) \gamma /(n+1) \lambda_{0}+1}\right. \\
& \cdot I_{(n-1) \gamma /(n+1) \lambda_{0}}\left(2 \sqrt{\frac{\alpha \sigma^{2}\left(\rho-\lambda_{0} t\right)}{(n+1) \lambda_{0}^{2}}}\right) \\
& \cdot \ln \frac{\left(2 \sqrt{\alpha \sigma^{2}\left(\rho-\lambda_{0} t\right) /(n+1) \lambda_{0}^{2}}\right)}{2}+\frac{1}{2}
\end{aligned}
$$




$$
\begin{aligned}
& \cdot \sum_{k=0}^{(n-1) \gamma /(n+1) \lambda_{0}-1} \frac{\left((n-1) \gamma /(n+1) \lambda_{0}-k-1\right) !}{k !} \\
& +\frac{1}{2}(-1)^{(n-1) \gamma /(n+1) \lambda_{0}} \\
& \cdot \sum_{k=0}^{\infty}\left(\frac{\left(2 \sqrt{\alpha \sigma^{2}\left(\rho-\lambda_{0} t\right) /(n+1) \lambda_{0}^{2}}\right)}{2}\right)^{2 k+(n-1) \gamma /(n+1) \lambda_{0}} \\
& \left.\left.\cdot \frac{\Psi(k+1)+\Psi\left((n-1) \gamma /(n+1) \lambda_{0}+k+1\right)}{k !\left((n-1) \gamma /(n+1) \lambda_{0}+k\right) !}\right]\right\},
\end{aligned}
$$

where $I_{\nu}(x)=\sum_{k=0}^{\infty}\left((x / 2)^{2 k+v} / k ! \Gamma(\nu+k+1)\right)$ and $\psi(x)$ is the logarithmic derivative of the gamma function.

Corollary 10. Under assumption of Corollary 9, let $n=2$. Then the form of $\sum(t)$ satisfies

$$
\begin{aligned}
& \left(\rho-\lambda_{0} t\right) \dot{\Sigma}(t)+\left(-\lambda_{0}+\frac{\gamma}{3 \lambda_{0}}\right) \dot{\sum}(t)-\frac{\alpha \sigma^{2}}{3 \lambda_{0}} \sum(t) \\
& \quad=0
\end{aligned}
$$

When $\gamma / 3 \lambda_{0}$ is not an integer, the solution of (55) is

$$
\begin{gathered}
\sum(t)=\left(2 \sqrt{\frac{\alpha \sigma^{2}\left(\rho-\lambda_{0} t\right)}{3 \lambda_{0}^{2}}}\right)^{\gamma / 3 \lambda_{0} / 2} \\
\cdot\left[C_{1} i^{\gamma / 3 \lambda_{0}} I_{\gamma / 3 \lambda_{0}}\left(2 \sqrt{\frac{\alpha \sigma^{2}\left(\rho-\lambda_{0} t\right)}{3 \lambda_{0}^{2}}}\right)\right. \\
+C_{2}\left(\left(i^{\gamma / 2 \lambda_{0}} I_{\gamma / 3 \lambda_{0}}\left(2 \sqrt{\frac{\alpha \sigma^{2}\left(\rho-\lambda_{0} t\right)}{3 \lambda_{0}^{2}}}\right) \cos \frac{\gamma \pi}{3 \lambda_{0}}\right.\right. \\
\left.-(-1)^{\gamma / 3 \lambda_{0}} i^{\gamma / 3 \lambda_{0}} I_{\gamma / 3 \lambda_{0}}\left(2 \sqrt{\frac{\alpha \sigma^{2}\left(\rho-\lambda_{0} t\right)}{3 \lambda_{0}^{2}}}\right)\right)
\end{gathered}
$$

$$
\left.\left.\cdot\left(\sin \frac{\gamma \pi}{3 \lambda_{0}}\right)^{-1}\right)\right]
$$

$$
\begin{aligned}
& \sum(t)=\left(2 \sqrt{\frac{\alpha \sigma^{2}\left(\rho-\lambda_{0} t\right)}{3 \lambda_{0}^{2}}}\right)^{\gamma / 3 \lambda_{0} / 2}\left\{C_{1} i^{\gamma / 3 \lambda_{0}} I_{\gamma / 3 \lambda_{0}}\left(2 \sqrt{\frac{\alpha \sigma^{2}\left(\rho-\lambda_{0} t\right)}{3 \lambda_{0}^{2}}}\right)+C_{2} i^{\gamma / 3 \lambda_{0}}\right. \\
& \quad \cdot \frac{2}{\pi}\left[(-1)^{\gamma / 3 \lambda_{0}+1} I_{\gamma / 3 \lambda_{0}}\left(2 \sqrt{\frac{\alpha \sigma^{2}\left(\rho-\lambda_{0} t\right)}{3 \lambda_{0}^{2}}}\right) \ln \frac{\left(2 \sqrt{\alpha \sigma^{2}\left(\rho-\lambda_{0} t\right) / 3 \lambda_{0}^{2}}\right)}{2}+\frac{1}{2} \sum_{k=0}^{\gamma / 3 \lambda_{0}-1} \frac{\left(\gamma / 3 \lambda_{0}-k-1\right) !}{k !}\right. \\
& \left.+\frac{1}{2}(-1)^{\gamma / 3 \lambda_{0}} \sum_{k=0}^{\infty}\left(\frac{\Psi(k+1)+\Psi\left(\gamma / 3 \lambda_{0}+k+1\right)}{k !\left(\gamma / 3 \lambda_{0}+k\right) !}\right]\right\}
\end{aligned}
$$

where $I_{\nu}(x)=\sum_{k=0}^{\infty}\left((x / 2)^{2 k+v} / \Gamma(k+1) \Gamma(\nu+k+1)\right)$ and $\psi(x)$ is the logarithmic derivative of the gamma function.

Proof. When $n=2$, from Corollary 9, (52) becomes

$$
\left(\rho-\lambda_{0} t\right) \ddot{y}+\left(-\lambda_{0}+\frac{\gamma}{3 \lambda_{0}}\right) \dot{y}-\frac{\alpha \sigma^{2}}{3 \lambda_{0}} y=0 .
$$

From the results of [27], when $\gamma / 3 \lambda_{0}$ is not an integer, the solution of (58) is

$$
y=\left(2 \sqrt{\frac{\alpha \sigma^{2}\left(\rho-\lambda_{0} t\right)}{3 \lambda_{0}^{2}}}\right)^{\gamma / 3 \lambda_{0} / 2}
$$

$$
\begin{aligned}
& {\left[C_{1} i^{\gamma / 3 \lambda_{0}} I_{\gamma / 3 \lambda_{0}}\left(2 \sqrt{\frac{\alpha \sigma^{2}\left(\rho-\lambda_{0} t\right)}{3 \lambda_{0}^{2}}}\right)\right.} \\
& +C_{2}\left(\left(i^{\gamma / 2 \lambda_{0}} I_{\gamma / 3 \lambda_{0}}\left(2 \sqrt{\frac{\alpha \sigma^{2}\left(\rho-\lambda_{0} t\right)}{3 \lambda_{0}^{2}}}\right) \cos \frac{\gamma \pi}{3 \lambda_{0}}\right.\right. \\
& \left.-(-1)^{\gamma / 3 \lambda_{0}} i^{\gamma / 3 \lambda_{0}} I_{\gamma / 3 \lambda_{0}}\left(2 \sqrt{\frac{\alpha \sigma^{2}\left(\rho-\lambda_{0} t\right)}{3 \lambda_{0}^{2}}}\right)\right) \\
& \left.\left.\cdot\left(\sin \frac{\gamma \pi}{3 \lambda_{0}}\right)^{-1}\right)\right] .
\end{aligned}
$$


When $\gamma / 3 \lambda_{0}$ is an integer, the solution of (58) is

$$
\begin{aligned}
& y=\left(2 \sqrt{\frac{\alpha \sigma^{2}\left(\rho-\lambda_{0} t\right)}{3 \lambda_{0}^{2}}}\right)^{\gamma / 3 \lambda_{0} / 2} \\
& \cdot\left\{C_{1} i^{\gamma / 3 \lambda_{0}} I_{\gamma / 3 \lambda_{0}}\left(2 \sqrt{\frac{\alpha \sigma^{2}\left(\rho-\lambda_{0} t\right)}{3 \lambda_{0}^{2}}}\right)+C_{2} i^{\gamma / 3 \lambda_{0}}\right. \\
& \cdot \frac{2}{\pi}\left[(-1)^{\gamma / 3 \lambda_{0}+1} I_{\gamma / 3 \lambda_{0}}\left(2 \sqrt{\frac{\alpha \sigma^{2}\left(\rho-\lambda_{0} t\right)}{3 \lambda_{0}^{2}}}\right)\right. \\
& \cdot \ln \frac{\left(2 \sqrt{\alpha \sigma^{2}\left(\rho-\lambda_{0} t\right) / 3 \lambda_{0}^{2}}\right)}{2}+\frac{1}{2} \\
& \cdot \sum_{k=0}^{\gamma / 3 \lambda_{0}-1} \frac{\left(\gamma / 3 \lambda_{0}-k-1\right) !}{k !}+\frac{1}{2}(-1)^{\gamma / 3 \lambda_{0}} \\
& \cdot \sum_{k=0}^{\infty}\left(\frac{\left(2 \sqrt{\alpha \sigma^{2}\left(\rho-\lambda_{0} t\right) / 3 \lambda_{0}^{2}}\right)}{2}\right)^{2 k+\gamma / 3 \lambda_{0}} \\
& \left.\left.\frac{\Psi(k+1)+\Psi\left(\gamma / 3 \lambda_{0}+k+1\right)}{k !\left(\gamma / 3 \lambda_{0}+k\right) !}\right]\right\}
\end{aligned}
$$

where $I_{\nu}(x)=\sum_{k=0}^{\infty}\left((x / 2)^{2 k+v} / \Gamma(k+1) \Gamma(\nu+k+1)\right)$ and $\psi(x)$ is the logarithmic derivative of the gamma function.

Corollary 11. Under assumption of Corollaries 8 and 9, the equilibrium strategy $\Delta(t)=X_{1}(t)-X_{2}(t)$ satisfies the form

$$
\left(\rho-\lambda_{0} t\right) \ddot{\Delta}(t)-\left(\lambda_{0}+\gamma\right) \dot{\Delta}(t)-\alpha \sigma^{2} \Delta(t)=0 .
$$

When $-\gamma / \lambda_{0}$ is not an integer, one has

$$
\begin{aligned}
& \Delta(t)=\left(2 \sqrt{\frac{\alpha \sigma^{2}\left(\rho-\lambda_{0} t\right)}{\lambda_{0}^{2}}}\right)^{-\gamma / 2 \lambda_{0}} \\
& \cdot\left[C_{1} i^{-\gamma / \lambda_{0}} \sum_{k=0}^{\infty} \frac{\left(\sqrt{\alpha \sigma^{2}\left(\rho-\lambda_{0} t\right)} / \lambda_{0}\right)}{\Gamma(k+1) \Gamma\left(-\gamma / \lambda_{0}+k+1\right)}\right. \\
& +C_{2}\left(\left(i^{-\gamma / \lambda_{0}} \sum_{k=0}^{\infty} \frac{\left(\sqrt{\alpha \sigma^{2}\left(\rho-\lambda_{0} t\right)} / \lambda_{0}\right)}{\Gamma(k+1) \Gamma\left(-\gamma / \lambda_{0}+k+1\right)}\right.\right. \\
& \cdot \cos \left(-\frac{\gamma}{\lambda_{0}} \pi\right)
\end{aligned}
$$

$$
\begin{aligned}
& \left.-i^{\gamma / \lambda_{0}} \sum_{k=0}^{\infty} \frac{\left(\sqrt{\alpha \sigma^{2}\left(\rho-\lambda_{0} t\right)} / \lambda_{0}\right)}{\Gamma(k+1) \Gamma\left(\gamma / \lambda_{0}+k+1\right)}\right) \\
& \left.\left.\cdot\left(\sin \left(-\frac{\gamma}{\lambda_{0}} \pi\right)\right)^{-1}\right)\right]
\end{aligned}
$$

When $-\gamma / \lambda_{0}$ is an integer, one gets

$$
\begin{aligned}
& \Delta(t)=\left(2 \sqrt{\frac{\alpha \sigma^{2}\left(\rho-\lambda_{0} t\right)}{\lambda_{0}^{2}}}\right)^{-\gamma / 2 \lambda_{0}} \\
& \left\{C_{1} i^{-\gamma / \lambda_{0}} \sum_{k=0}^{\infty} \frac{\left(\sqrt{\alpha \sigma^{2}\left(\rho-\lambda_{0} t\right)} / \lambda_{0}\right)}{\Gamma(k+1) \Gamma\left(-\gamma / \lambda_{0}+k+1\right)}\right. \\
& +C_{2} i^{-\gamma / \lambda_{0}}\left[(-1)^{\gamma / \lambda_{0}}\right. \\
& \cdot \sum_{k=0}^{\infty} \frac{\left(\sqrt{\alpha \sigma^{2}\left(\rho-\lambda_{0} t\right)} / \lambda_{0}\right)}{\Gamma(k+1) \Gamma\left(-\gamma / \lambda_{0}+k+1\right)} \\
& \cdot \ln \frac{\sqrt{\alpha \sigma^{2}\left(\rho-\lambda_{0} t\right)}}{\lambda_{0}}+\frac{1}{2} \sum_{k=0}^{\gamma / \lambda_{0}-1}(-1)^{k} \\
& \cdot\left(\frac{\sqrt{\alpha \sigma^{2}\left(\rho-\lambda_{0} t\right)}}{\lambda_{0}}\right)^{2 k-\gamma / \lambda_{0}} \frac{\left(\gamma / \lambda_{0}-k-1\right) !}{k !} \\
& +\frac{1}{2}(-1)^{\gamma / \lambda_{0}} \sum_{k=0}^{\infty}\left(\frac{\sqrt{\alpha \sigma^{2}\left(\rho-\lambda_{0} t\right)}}{\lambda_{0}}\right)^{2 k+\gamma / \lambda_{0}} \\
& \left.\left.\frac{\psi\left(\gamma / \lambda_{0}+k+1\right)+\psi(k+1)}{k !\left(\gamma / \lambda_{0}+k\right) !}\right]\right\} \text {. }
\end{aligned}
$$

Proof. Since $\Delta(t)=X_{1}(t)-X_{2}(t),(46)$ becomes

$$
\alpha \sigma^{2} \Delta(t)-\dot{\lambda}(t) \dot{\Delta}(t)+\gamma \dot{\Delta}(t)-\lambda(t) \ddot{\Delta}(t)=0 .
$$

Let $y=\Delta(t)$ and $\lambda(t)=\rho-\lambda_{0} t$; (64) becomes

$$
\left(\rho-\lambda_{0} t\right) \ddot{y}-\left(\lambda_{0}+\gamma\right) \dot{y}-\alpha \sigma^{2} y=0 .
$$


From the results of [27], we get the solution of (65) in the following form. When $-\gamma / \lambda_{0}$ is not an integer, one has

$$
\begin{aligned}
y & =\left(2 \sqrt{\frac{\alpha \sigma^{2}\left(\rho-\lambda_{0} t\right)}{\lambda_{0}^{2}}}\right)^{-\gamma / 2 \lambda_{0}} \\
& \cdot\left[C_{1} i^{-\gamma / \lambda_{0}} \sum_{k=0}^{\infty} \frac{\left(\sqrt{\alpha \sigma^{2}\left(\rho-\lambda_{0} t\right)} / \lambda_{0}\right)}{\Gamma(k+1) \Gamma\left(-\gamma / \lambda_{0}+k+1\right)}\right. \\
& +C_{2}\left(i^{-\gamma / \lambda_{0}} \sum_{k=0}^{\infty} \frac{\left(\sqrt{\alpha \sigma^{2}\left(\rho-\lambda_{0} t\right)} / \lambda_{0}\right)}{\Gamma+1) \Gamma\left(-\gamma / \lambda_{0}+k+1\right)}\right. \\
& \cdot \cos \left(-\frac{\gamma}{\lambda_{0}} \pi\right) \\
& \left.-i^{\gamma / \lambda} \sum_{k=0}^{\infty} \frac{\left(\sqrt{\left.\alpha \sigma^{2}\left(\rho-\lambda_{0} t\right) / \lambda_{0}\right)}\right.}{\Gamma+1) \Gamma\left(\gamma / \lambda_{0}+k+1\right)}\right) \\
& \left.\left.\cdot\left(\sin \left(-\frac{\gamma}{\lambda_{0}} \pi\right)\right)^{-1}\right)\right] .
\end{aligned}
$$

When $-\gamma / \lambda_{0}$ is an integer, we have

$$
\begin{aligned}
& y=\left(2 \sqrt{\frac{\alpha \sigma^{2}\left(\rho-\lambda_{0} t\right)}{\lambda_{0}^{2}}}\right)^{-\gamma / 2 \lambda_{0}} \\
& \cdot\left\{C_{1} i^{-\gamma / \lambda_{0}} \sum_{k=0}^{\infty} \frac{\left(\sqrt{\alpha \sigma^{2}\left(\rho-\lambda_{0} t\right)} / \lambda_{0}\right)}{\Gamma(k+1) \Gamma\left(-\gamma / \lambda_{0}+k+1\right)}\right. \\
& +C_{2} i^{-\gamma / \lambda_{0}}\left[(-1)^{\gamma / \lambda_{0}}\right. \\
& \cdot \sum_{k=0}^{\infty} \frac{\left(\sqrt{\alpha \sigma^{2}\left(\rho-\lambda_{0} t\right)} / \lambda_{0}\right)}{\Gamma(k+1) \Gamma\left(-\gamma / \lambda_{0}+k+1\right)} \\
& \cdot \ln \frac{\sqrt{\alpha \sigma^{2}\left(\rho-\lambda_{0} t\right)}}{\lambda_{0}}+\frac{1}{2} \sum_{k=0}^{\gamma / \lambda_{0}-1}(-1)^{k} \\
& \cdot\left(\frac{\sqrt{\alpha \sigma^{2}\left(\rho-\lambda_{0} t\right)}}{\lambda_{0}}\right)^{2 k-\gamma / \lambda_{0}} \frac{\left(\gamma / \lambda_{0}-k-1\right) !}{k !}
\end{aligned}
$$

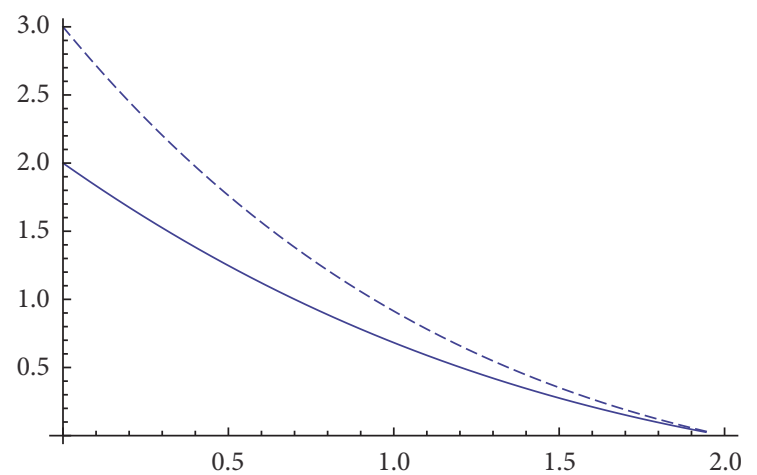

Figure $1: \alpha=1 ; \sigma=1 ; \rho=1 ; \lambda_{0}=0.2 ; \gamma=0.3 ; X_{1}(0)=3$ (dashed); $X_{2}(0)=2$ (solid).

$$
\begin{aligned}
& +\frac{1}{2}(-1)^{\gamma / \lambda_{0}} \sum_{k=0}^{\infty}\left(\frac{\sqrt{\alpha \sigma^{2}\left(\rho-\lambda_{0} t\right)}}{\lambda_{0}}\right)^{2 k+\gamma / \lambda_{0}} \\
& \left.\left.\cdot \frac{\psi\left(\gamma / \lambda_{0}+k+1\right)+\psi(k+1)}{k !\left(\gamma / \lambda_{0}+k\right) !}\right]\right\}
\end{aligned}
$$

where $\Delta(0)=x_{1}-x_{2}$ and $\Delta(T)=0$.

Corollary 12. Under assumption of Corollaries 10 and 11, the equilibrium strategies $X_{1}^{*}(t)$ and $X_{2}^{*}(t)$ are $X_{1}^{*}(t)=\left(\Sigma^{*}(t)+\right.$ $\left.\Delta^{*}(t)\right) / 2$ and $X_{2}^{*}(t)=\left(\Delta^{*}(t)-\sum^{*}(t)\right) / 2$, respectively.

Proof. From Corollaries 10 and 11, $\sum^{*}(t)=X_{1}^{*}(t)+X_{2}^{*}(t)$ and $\Delta(t)=X_{1}^{*}(t)-X_{2}^{*}(t)$; we have $X_{1}^{*}(t)=\left(\sum^{*}(t)+\Delta^{*}(t)\right) / 2$ and $X_{2}^{*}(t)=\left(\Delta^{*}(t)-\sum^{*}(t)\right) / 2$.

\section{Conclusion}

The existence and uniqueness of Nash equilibrium are proven in the previous sections. We get the concrete form of an agent's optimal strategy in a special case. Next, we will discuss the variation of strategy when market volatility and the coefficient of temporary market impact change.

From Figures 1-3, we get certain information. When $\sigma$ increases from 0.5 to 1.5 , agent 1 realizes that the market volatility risk is increasing. The agent's revenue will have a bigger fluctuation following the market volatility. Therefore, the agent will speed up liquidation in the former half of the time $[0, T]$ so that the agent can get more revenue. At the same time, he will decrease liquidation so that the cost decreases. From Figure 3, it shows that the trading speed increases following the increasing of coefficient $\lambda_{0}$. When the coefficient $\lambda_{0}$ increases, the influence of the temporary impact will decrease. The trading cost will decrease so that the agent gets the maximization of revenue. When the coefficient of temporary market impact decreases with time, the agent realizes that the influence of temporary impact is decreasing and slows down the trading speed. 


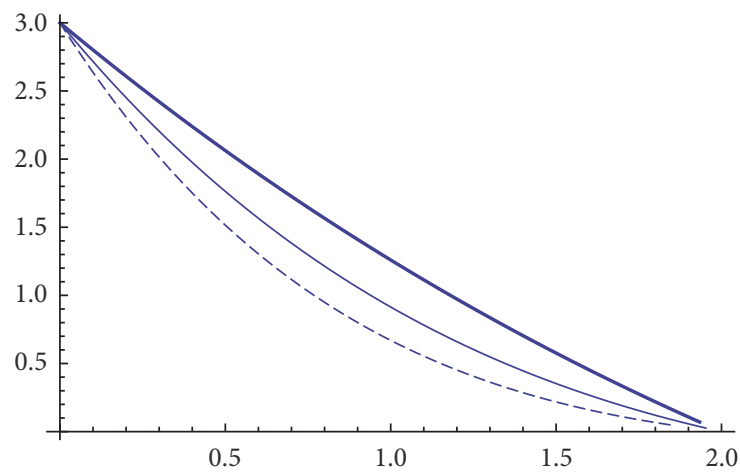

Figure $2: \alpha=1 ; \sigma_{1}=1.5$ (dashed); $\sigma_{2}=1$ (solid); $\sigma_{3}=0.5$ (thick); $\rho=1 ; \lambda_{0}=0.2 ; \gamma=0.3 ; X_{1}(0)=3 ; X_{2}(0)=2$.

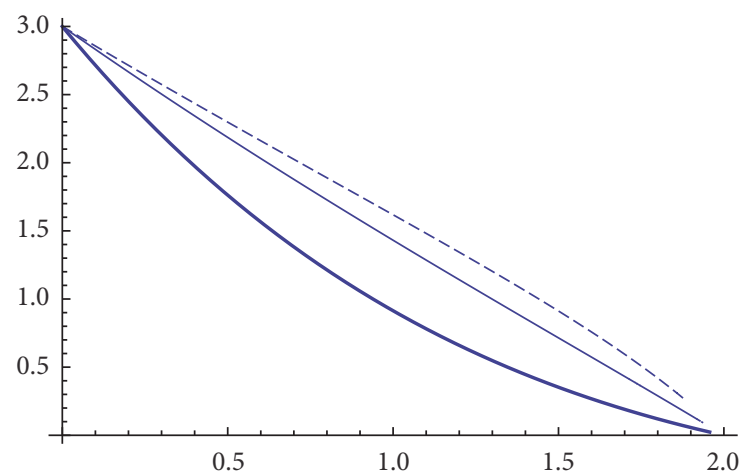

Figure 3: $\alpha=1 ; \sigma_{3}=0.5 ; \rho=1 ; \lambda_{01}=0.2$ (thick); $\lambda_{02}=0.34$ (solid); $\lambda_{03}=0.44$ (dashed), $\gamma=0.3 ; X_{1}(0)=3 ; X_{2}(0)=2$.

\section{Conflicts of Interest}

The authors declare that there are no conflicts of interest regarding the publication of this paper.

\section{References}

[1] H. Markowitz, "Portfolio selection," The Journal of Finance, vol. 7, no. 1, pp. 77-91, 1952.

[2] H. Markowitz, Portfolio Selection: Efficient Diversification of Investments, John Wiley \& Sons, New York, NY, USA, 1959.

[3] P. A. Samuelson, "Lifetime portfolio selection by dynamic stochastic programming," The Review of Economics and Statistics, vol. 51, no. 3, pp. 239-246, 1969.

[4] N.H. Hakansson, "Multi-period mean-variance analysis: toward a general theory of portfolio choice," The Journal of Finance, vol. 26, no. 4, pp. 857-884, 1971.

[5] S. R. Pliska, Introduction to Mathematical Finance, Blackwell, Malden, Mass, USA, 1997.

[6] J. C. Cox and C.-f. Huang, "Optimal consumption and portfolio policies when asset prices follow a diffusion process," Journal of Economic Theory, vol. 49, no. 1, pp. 33-83, 1989.

[7] D. Duffie and H. R. Richardson, "Mean-variance hedging in continuous time," The Annals of Applied Probability, vol. 1, no. 1, pp. 1-15, 1991.
[8] I. Karatzas, J. P. Lehoczky, and S. E. Shreve, "Optimal portfolio and consumption decisions for a "small investor" on a finite horizon," SIAM Journal on Control and Optimization, vol. 25, no. 6, pp. 1557-1586, 1987.

[9] M. Schweizer, "Approximation pricing and the varianceoptimal martingale measure," The Annals of Probability, vol. 24, no. 1, pp. 206-236, 1996.

[10] R. Almgren and N. Chriss, "Optimal execution of portfolio transactions," The Journal of Risk, vol. 3, no. 2, pp. 5-39, 2001.

[11] R. F. Almgren, "Optimal execution with nonlinear impact functions and trading-enhanced risk," Applied Mathematical Finance, vol. 10, no. 1, pp. 1-18, 2003.

[12] G. Huberman and W. Stanzl, "Price manipulation and quasiarbitrage," Econometrica, vol. 72, no. 4, pp. 1247-1275, 2004.

[13] J.-P. Bouchaud, Y. Gefen, M. Potters, and M. Wyart, "Fluctuations and response in financial markets: the subtle nature of 'random' price changes," Quantitative Finance, vol. 4, no. 2, pp. 176-190, 2004.

[14] M. Attari, A. S. Mello, and M. E. Ruckes, "Arbitraging arbitrageurs," Journal of Finance, vol. 60, no. 5, pp. 2471-2511, 2005.

[15] T. Schoeneborn and A. Schied, "Liquidation in the face of adversity: stealth vs. sunshine trading, predatory trading vs. liquidity provision," Munich Personal Repec Archive, 2007.

[16] B. I. Carlin, M. S. Lobo, and S. Viswanathan, "Episodic liquidity crises: cooperative and predatory trading," Journal of Finance, vol. 62, no. 5, pp. 2235-2274, 2007.

[17] A. Schied and T. Schoeneborn, "Risk aversion and the dynamics of optimal trading strategies in illiquid markets," Munich Personal RePEc Archive, vol. 13, pp. 181-204, 2009.

[18] R. A. Carmona and Z. Joseph Yang, "Predatory trading: a game on volatility and liquidity," Quantitative Finance, 2008, http:// www.princeton.edu/ rcarmona/download/fe/PredatoryTradingGameQF.pdf.

[19] A. Schied, T. Schoneborn, and M. Tehranchi, "Optimal basket liquidation for CARA investors is deterministic," Applied Mathematical Finance, vol. 17, no. 6, pp. 471-489, 2010.

[20] J. Lorenz and R. Almgren, "Mean-variance optimal adaptive execution," Applied Mathematical Finance, vol. 18, no. 5, pp. 395-422, 2011.

[21] J. Gatheral, "No-dynamic-arbitrage and market impact," Quantitative Finance, vol. 10, no. 7, pp. 749-759, 2010.

[22] A. Schied, "Order book resilience, price manipulation, and the positive portfolio problem," 2010, http://helper.ipam.ucla.edu/ publications/fin2010/fin2010_8956.pdf.

[23] A. Schied and T. Zhang, "A state-constrained differential game arising in optimal portfolio liquidation," Mathematical Finance, 2015.

[24] L. Bachelier, “Thorie de la spculation," Annales Scientifiques de l'École Normale Supérieure, vol. 3, no. 17, pp. 21-86, 1900.

[25] D. Bertsimas and A. W. Lo, "Optimal control of execution costs," Journal of Financial Markets, vol. 1, no. 1, pp. 1-50, 1998.

[26] Northeast Normal University, Ordinary Differential Equation, Higher Education Press, Beijing, China, 2015.

[27] D. P. Andrei and F. Z. Valentin, Handbook of Exact Solutions for Ordinary Differential Equations, Chapman Hall/CRC, 2003. 


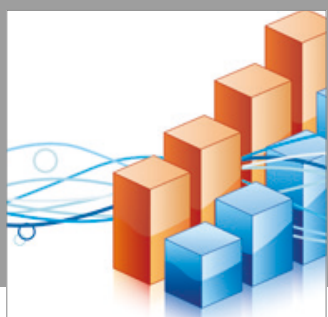

Advances in

Operations Research

vatersals

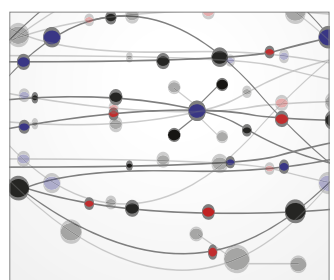

\section{The Scientific} World Journal
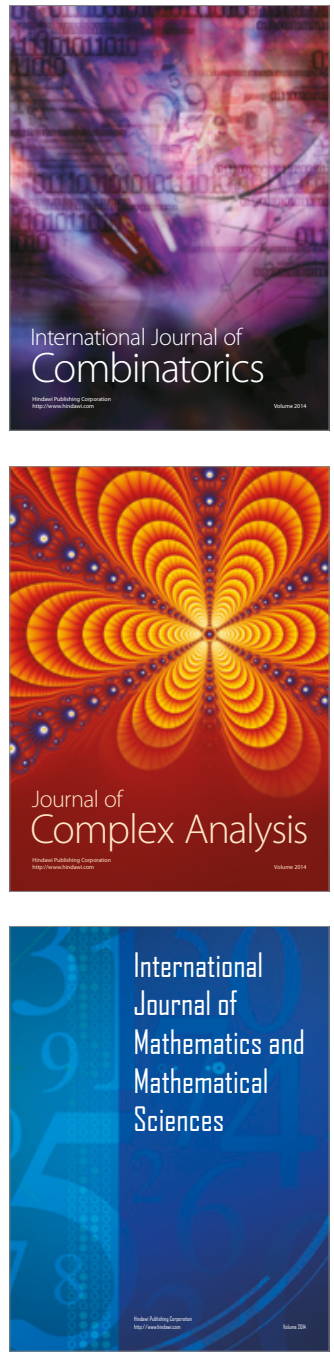
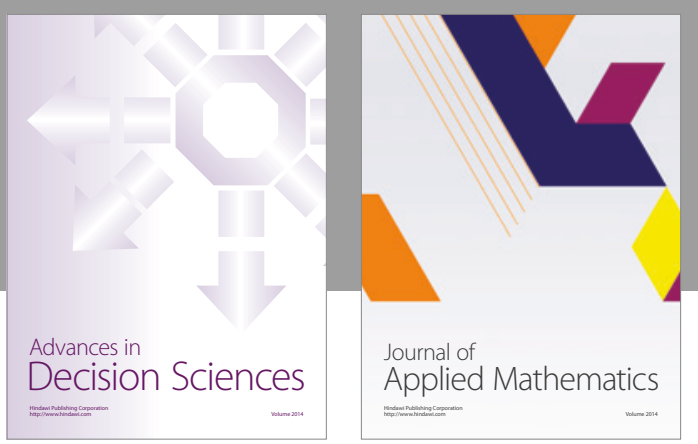

Algebra

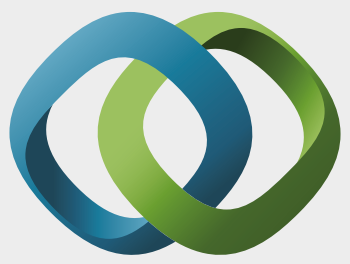

\section{Hindawi}

Submit your manuscripts at

https://www.hindawi.com
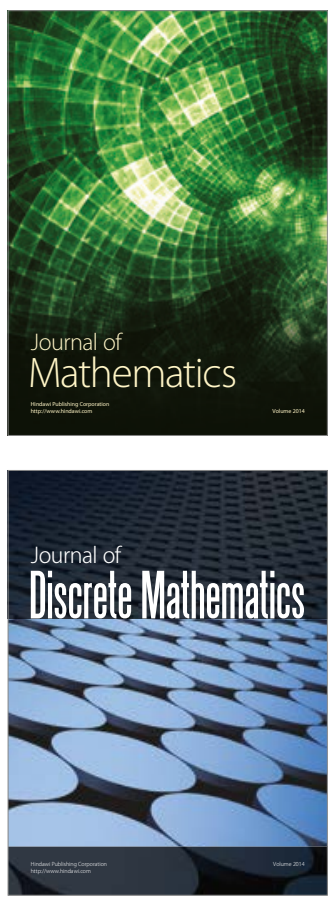

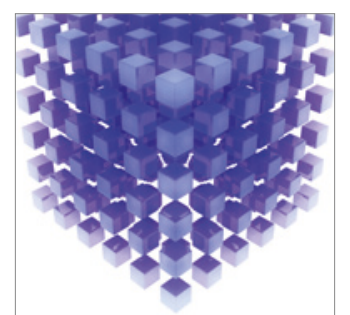

Mathematical Problems in Engineering
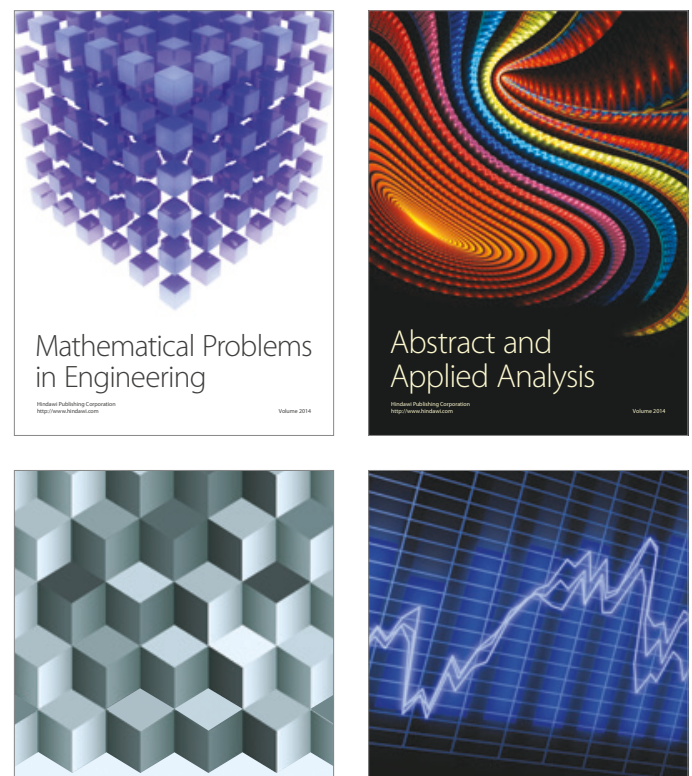

Journal of

Function Spaces

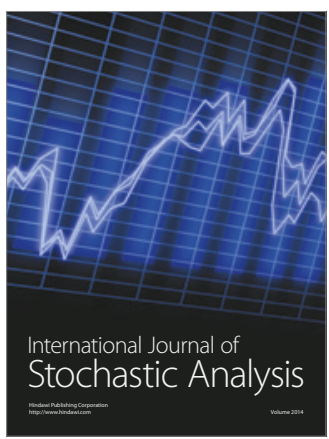

Probability and Statistics
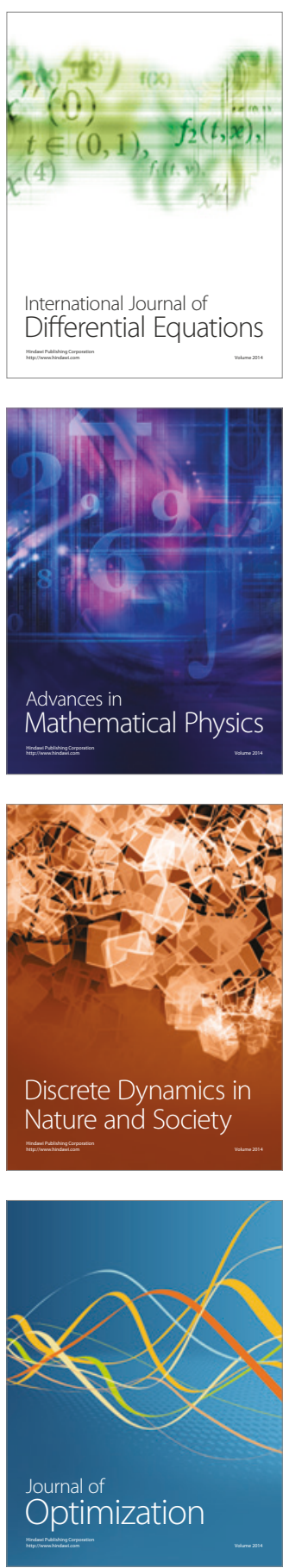